Sociologie et sociétés

\title{
Le sentiment d'aliénation comme opérateur de mouvance : réflexion à partir d'expériences de vie de jeunes en situation précaire
}

\section{Paul Grell}

Volume 34, numéro 1, printemps 2002

La théorie du choix rationnel contre les sciences sociales? Bilan des débats contemporains

URI : https://id.erudit.org/iderudit/009759ar

DOI : https://doi.org/10.7202/009759ar

Aller au sommaire du numéro

Éditeur(s)

Les Presses de l'Université de Montréal

ISSN

0038-030X (imprimé)

1492-1375 (numérique)

Découvrir la revue

Citer cet article

Grell, P. (2002). Le sentiment d'aliénation comme opérateur de mouvance :

réflexion à partir d'expériences de vie de jeunes en situation précaire.

Sociologie et sociétés, 34(1), 199-214. https://doi.org/10.7202/009759ar 


\section{Le sentiment d'aliénation comme opérateur de mouvance : réflexion à partir d'expériences de vie de jeunes en situation précaire}

\section{PAUL GRELL}

École de travail social

Université de Moncton

Moncton (Nouveau-Brunswick), Canada $\mathrm{E}_{1} \mathrm{~A}_{3} \mathrm{~V}_{5}$

Courriel : grellp@umoncton.ca

$\mathrm{O}$ N NE PARLE PLUS BEAUCOUP d'aliénation à l'heure actuelle, pour deux raisons sans doute. D'une part, les essais de conceptualisation, notamment dans la perspective des grands récits de légitimation, se trouvent affectés d'un certain coefficient de scepticisme, même si ceux-ci se fondent de toute évidence, comme dans le cas de l'aliénation, sur des observations que tout le monde peut faire (atomisation, non-sens, impuissance, ennui, exploitation, etc.). Et d'autre part, cette notion est déchirée entre des concepts aussi différents que ceux de l'action, du sens, du sentiment, etc., allant des troubles pathologiques aux dislocations sociales à grande échelle et cela, au détriment d'une certaine unité théorique.

Cet article sera essentiellement consacré à une réflexion sur le sentiment d'aliénation en tant que tel, comme opérateur de mouvance ${ }^{1}$, dans le sens où la conscience qui l'habite est déjà le signe, l'indication d'une désaliénation possible. Cet article s'articulera en quatre parties. Il s'agira d'abord de spécifier les termes dans lesquels cette notion d'aliénation est utilisée, d'en préciser au départ le contexte de réflexion avant de proposer, en s'inspirant d'une enquête de terrain, le portrait des pratiques et sensibilités

1. Je tiens à remercier les évaluateurs de cet article pour leurs précieux commentaires critiques qui m'ont aidé à en clarifier certains aspects. Ce travail s'inscrit dans le cadre d'un programme de recherche financé par le CRSH. Je remercie le Conseil pour l'aide accordée. 
de jeunes en situation précaire. Ensuite, il s'agira de faire voir l'importance de la question du «comment lutter contre le sentiment d'aliénation?» en indiquant, à tout le moins sous forme d'esquisse, la réponse qui en est donnée.

\section{DE L'ALIÉNATION AU SENTIMENT D'ALIÉNATION}

Il convient de remarquer dès l'abord le caractère polysémique de la notion d'aliénation. À son propos, Paul Ricœur parle de «mot-hôpital» dans lequel tous les malaises viennent se coucher (1974, p. 661). Dans le débat sociologique, cette notion tend à prendre pour base l'état des relations qu'entretient le travailleur avec le produit de son travail et avec les institutions qui en disposent et le déterminent. Elle désigne la situation dans laquelle le travailleur est dessaisi par (et pour) un autre. La discussion porte surtout sur la validité théorique de la notion et sur la manière de l'ériger éventuellement en concept rigoureux (Seeman, Vidal, Amiot et Touraine, 1967). Ce débat relève d'une attitude contemplative se bornant à constater tantôt des situations aux composantes aliénantes (ou anomiques), tantôt des sujets pris du «malaise global de la personnalité» (Vidal, 1969).

À l'inverse, cette notion tend à prendre une base différente chez des auteurs comme Castoriadis, par exemple, où elle se réfere à la vie comme processus de création largement indéterminé dont le moteur est l'autonomie. «L'être comme il dit est à être». Ce commentaire de Diane Pacom, insiste sur ce qui est au centre de l'œuvre de Castoriadis, à savoir que «la vie est toujours un processus, et que l'individu responsable [...] est un individu qui se laisse travailler par la vie» (Pacom, 1999, p. 177). Au niveau individuel, l'aliénation est à l'opposé de l'autonomie et désigne la législation ou la régulation du sujet par le discours de l'autre : «c'est que, dominé par ce discours, le sujet se prend pour quelque chose qu'il n'est pas» (Castoriadis, 1975, p. 140). Sa caractéristique, du point de vue qui intéresse ici, est son rapport à l'imaginaire : «Le sujet est dominé par un imaginaire vécu comme plus réel que le réel [...] qui s’est arrogé la fonction de définir pour le sujet et la réalité et son désir» (Ibid., p. 41). Mais l'aliénation trouve ses conditions, audelà du «discours de l'autre», au-delà «de la perte de soi dans l'autre», dans la structure oppressive de la société : «dans l'anonymat collectif, l’impersonnalité des mécanismes économiques du marché ou de la rationalité du Plan, de la loi de quelques-uns présentée comme la loi tout court» (Ibid., p. 149). C'est ce que Castoriadis appelle l'hétéronomie sociale : l'autre est «incarné» ailleurs que dans l'inconscient individuel. Il est incarné dans des «lois» qui ne sont pas seulement juridiques, mais des lois sociales, des codes, des représentations, des structures et des institutions sociales qui agissent comme des «mitraillettes», des «ordres de mobilisation», des «prisons», etc. Ces expressions montrent combien puissant peut être le dessaisissement d'être dans les conditionnements effectués par ces «lois», combien profonde l'aliénation de la société à ses institutions.

Nous visons ce fait $[\ldots]$ que l'institution une fois posée, semble s'autonomiser, qu'elle possède son inertie et sa logique propre, qu'elle dépasse, dans sa survie et dans ses effets, sa fonction, ses «fins» et ses «raisons d'être». Les évidences se renversent; ce qui pouvait être vu «au départ» comme un ensemble d'institutions au service de la société, devient une société au service des institutions (Ibid., p. 151). 
En conséquence, la discussion porte surtout sur le développement et l'exercice de l'autonomie comme autant de moments d'un processus d'élucidation et de transformation de la réalité. L'attitude est ici foncièrement politique et constate que l'aliénation ne se confond pas simplement avec des dysfonctionnements, syndromes, exclusions, etc., mais qu'elle est liée pour une très grosse part à toute la structure sociale et que des novations radicales sont nécessaires pour que cela change.

Dans cette réflexion qui s'inspire de récits d'expérience de jeunes en situation précaire, c'est l'attitude notionnelle qui prévaut (Grell et Wery, 1993, p. 169). Celle-ci procède par approches concentriques et prend acte de l'hétérogénéité du monde : « [cette attitude] donne sur un même objet des éclairages divers, elle indique qu'il est à la fois ceci et cela» (Maffesoli, 1985, p. 51). Parler de «sentiment d'aliénation » participe de cette attitude. Le mot sentiment renvoie à un vécu d'ordre global.

Dans la langue classique, il a toujours dénoté autre chose que l'émotion ou la passion. C'est en ce sens qu'on parlait du «sentiment religieux», du «sentiment esthétique», en désignant un type de sensibilité à certaines choses qui avait un caractère global (Ledrut, 1979, p. 81).

Ce caractère global nous conduit à poser le problème de la totalité sociale et à définir le sentiment d'aliénation comme une forme sociale de rupture, concrète et sensible, se manifestant par des obstacles, des épreuves, dont la définition ne peut être faite hors du sens qui est donné dans la vie qui travaille les êtres humains et entrave leur capacité d'autoproduction et d'autoorganisation. Que le terme d'aliénation désigne des situations sociales dans lesquelles individus et collectivités sont dessaisis de leur activité et du sens de leur action et/ou qu'il désigne des lois et des institutions par lesquels ils rencontrent des obstacles constamment renouvelés dès qu'ils cherchent à se déployer et à exister socialement, ce sentiment d'aliénation ne peut être défini indépendamment de la conscience qui en est progressivement prise. C'est la progression de la recherche sur le terrain, avec ses connexions, confrontations, «soudures imprévues», entre savoirs locaux et catégories conceptuelles, qui permettra d'en prendre toute l'ampleur : «l'aller-retour dialectique continu entre le plus local des détails locaux et la plus globale des structures globales» dirait Clifford Geertz (1986, p. 88).

Dans cet article, la notion de sentiment d'aliénation est vue comme une sorte de type idéal servant à « déceler et analyser les relations qui peuvent exister» (Weber, 1964, p. 318) en rapport avec les expériences que l'ont fait, non dans la perspective d'arriver à définir l'aliénation comme concept, mais dans celle d'appréhender ce que chacun imagine, construit pour soi comme aliéné et inaliénable. S’inscrivant dans la logique d'une société invivable, ce sentiment ne cesse d'être ambigu : à la fois expérience proche de ce que Hannah Arendt nomme la «désolation», définissant celle-ci comme «expérience d'absolue non-appartenance au monde» (Chaumont, 1991, p. 37) mais aussi forme en creux d'une possible émancipation dans la recherche de simplicité, de déraison même, «avec ses énoncés paradoxaux : perdre pour recevoir, sortir pour se retrouver...»(Cingolani, 1994, p. 102). Il est à la fois expérience non voulue et intense «espérance que quelque chose se gagne en échange de ce qui se perd, que quelque chose se fait à travers ce qui se défait» (Ricœur, 1974, p. 661). 


\section{CONTEXTE DE LA RÉfLEXION : DES JEUNES EN SITUATION}

Amérique du Nord, Canada, côte est du Nouveau-Brunswick, Acadie, monde précaire, jeunes, faible scolarité... autant de mots clés pour situer le terrain de réflexion. En arrière-fond : la difficulté pour beaucoup de jeunes de mener « une petite vie» dans un monde qui se présente à eux comme de plus en plus précaire.

Au départ, une littérature abondante qui offre deux représentations séparées du sujet. La première introduit la notion de jeunes travailleurs précaires pour spécifier le rapport négatif qu'une catégorie sociale (les «jeunes précaires» en l'occurrence) entretient avec le travail intermittent. Cette notion renvoie «à ces emplois de courte durée, à temps partiel non volontaire, faiblement rémunérés et sans avantages sociaux» (Gauthier, 1996, p. 19) se prolongeant dans différents statuts allant de "chômeur régulier» à «sans-statut» (Gauthier et Mercier, 1994; Schnapper, 1989). La deuxième élargit cette notion dans le sens de la précarité d'existence, c'est-à-dire : de jeunes confrontés à un monde où rien ne va plus de soi (Grell, 1999; Zoll, 1992). Il revient à Patrick Cingolani (1986) d'avoir attiré l'attention sur l'impasse que faisait ainsi la deuxième représentation sur la première dans sa tentative de sortir de l'unité de signification incarnée par la centralité du travail salarié (le «hors du travail salarié point de salut»). Bien que cette distinction ne soit pas antinomique, elle s'accompagne de questionnements, voire de schèmes d'intelligibilité (Berthelot, 1990), souvent très différents.

Selon les premiers schèmes d'intelligibilité, souvent en référence avec l'ancien institué (la génération du baby boom), les questions tournent autour des modèles d'entrée dans la vie (Galland, 1984; 1990) où le «devenir adulte» est nécessairement associé à (passe par) la centralité du travail salarié. Dans les autres schèmes, dont les auteurs sont rarement les mêmes, les questions pointent plutôt «l'espace social précaire» (René, 1993) où le «rester jeune» fait partie intégrante de pratiques polymorphes (Schehr, 1999). Les uns et les autres - l'exposé est schématique — débattent pour savoir si les jeunes précaires sont des ratés du système ou, au contraire, sont en train de créer de nouveaux modes de vie, des victimes ou des mutants effectifs. Mais, dans ce genre de débat, comme le constate Norbert Elias (1991, p. 132), «on est souvent poussé à prêter une plus grande réalité à ce à quoi on accorde une plus grande valeur» : les uns considèrent les sociétés comme plus réelles et plus importantes que les individus qui les constituent, tandis que les autres croient au contraire constater que les individus pris isolément sont la réalité.

Dans un camp comme dans l'autre se confond au niveau de la conscience ce dont on pense que ce devrait être avec l'observation de ce qui est; étant donné la violence des troubles auxquels se trouvent exposés, dans le champ de force des tensions politiques et sociales, les tenants de ces convictions sociales opposées, ce sont les premiers qui l'emportent habituellement (Ibid., p. 127).

Comment faire coïncider ces deux représentations ou du moins réduire le gouffre qu'elles établissent entre individu et société, alors même que nous reconnaissons la plupart du temps qu'il s'agit d'un faux problème. En effet, comme le constate encore Elias :

Toute société humaine se compose d'individus isolés et tout individu humain n'est véritablement humain qu'à partir du moment où il apprend à agir, à parler et à exercer sa 
sensibilité dans la société des autres. La société sans individus et l'individu sans société sont des choses qui n'existent pas (Ibid., p. 117).

C'est pourtant dans ces termes que la question reste souvent posée : une société des jeunes? Une société sans les jeunes? Etc. (Dumont, 1986; Gauthier, 1994). En fait, en chargeant ces mots d'impressions et d'appréciations affectives différentes, nous nous empêchons de voir qu'il y a là deux aspects d'une seule et même configuration sociale dont le sentiment (notamment le sentiment d'aliénation) et l'expression dans des formes (des structures) constituent deux faces du même : la recherche d'autonomie (Singly, 2000).

Dans un tel contexte, poser la question $\mathrm{du}$ «comment lutter, dans la vie quotidienne, contre le sentiment d'aliénation? », c'est en quelque sorte mailler d'emblée les individus et la société comme par contraste (conflits) entre des niveaux d'indépendance (individualisation) et de multiples liens d'interdépendance réciproque : les deux aspects d'une seule et même réaction humaine. D’une part, la possibilité de décider pour soi-même de façon autonome (le désir d'être soi-même), d'autre part, l'impossibilité de décider pour soi-même de façon autonome (le désir d'être intégré).

Partant de ce besoin d'autonomie qui va de pair avec celui d'appartenance, la réflexion dont il est question s'appuie sur une vaste enquête, menée sur la côte est du Nouveau-Brunswick entre 1993 et 1995 (voir annexe), portant sur une catégorie de jeunes dont on pouvait raisonnablement penser qu'elle était plus sujet à une existence aliénée. Cette enquête avait pour point de départ la question suivante : "Quelles sont les compétences autour desquelles s'organise progressivement l'entrée des jeunes dans le monde précaire leur permettant une maîtrise suffisamment affirmée pour infléchir leur itinéraire dans le sens qu'ils souhaitent?» (Grell, 1999, p. 12). Les jeunes qui ont participé à cette recherche sont tous sortis du secondaire avec un bagage scolaire plus ou moins faible (des cours de niveau modifié en mathématique et/ou en français). L'enquête portait sur celles et ceux dont on peut penser a priori que les conditions d'entrée dans le monde apparaissent moins assurées, plus étriquées. L'optique choisie n'est pas d'abord fonctionnelle et pragmatique et ce, pour deux raisons : 1) les problèmes auxquels les jeunes sont confrontés, comme nous tous d'ailleurs, exigent plus que des recettes; 2) réduire ces problèmes aux caractéristiques apparentes de ceux qui les vivent plus douloureusement que d'autres, c'est faire fi de la réalité. Nous n'avons pas voulu partir d'un ensemble de caractéristiques pour déterminer des profils cibles correspondant à des problèmes particuliers et des situations à risque. Par exemple, il aurait été très simple, à partir des $6 \%$ de jeunes de l'échantillon qui n'ont jamais eu d'emploi, de déterminer que telle ou telle catégorie a plus de chance (on de risque) d'être dans ce cas. Dans une optique de ce genre, on ne tient absolument pas compte de la réalité telle qu'elle est et telle qu'elle est réellement vécue sur le terrain. Par exemple, parmi ces jeunes qui n'ont jamais eu d'emploi, certains ont un hobby qu'ils maîtrisent très bien. Ils le maîtrisent si bien qu'on s'adresse à eux pour se procurer leurs œuvres. N'est-ce pas biaiser la situation que de les réduire à une absence de fonctionnalité face à l'emploi? 


\section{PORTRAIT DES PRATIQUES ET SENSIBILITÉS SOCIALES}

L'optique choisie ici est une optique phénoménologique reconnaissant au fondement de la vie sociale l'importance des déplacements de sens où les choses de la vie sont investies d'autres significations que leurs significations autorisées et les gestes quotidiens des signes créateurs qui renvoient à l'imaginaire social. Dans cette optique, il s'agit d'atteindre la base symbolique des pratiques, avec notamment les détours que les jeunes font pour vivre et agir. Pour les jeunes d'aujourd'hui, confrontés à un monde précaire, la question n'est pas seulement de savoir comment y être fonctionnel, mais de comprendre - et cela signifie évidemment : d'agir sur, de résister à — l'impuissance existentielle de fait incompréhensible, mais incontournable à force d'évidence violente, du monde dans lequel ils se trouvent. Il y a là un double mouvement. Tout se passe comme s'ils étaient au plein milieu d'un tournant à partir desquelles devront s'inventer d'autres voies qu'ils pressentent obscurément. Il s'agit, chez plusieurs d'entre eux, d'une sensibilité sociale aiguë — mélange de représentation, de sentiment et d'action — qui donne forme à la réalité du monde à travers les expériences qu'ils en font, la conscience progressive qu'ils en prennent et la quête de sens qu'ils entreprennent. Nous tentons ici, à grands traits, d'en esquisser le portrait vivant, filmé.

\section{Un travail attirant qui s'avère souvent pénible («mortel »)}

Les jeunes qui ont un emploi ${ }^{2}$ travaillent fort, mais les salaires sont bas. Ils permettent difficilement de vivre, de fonder une famille, de s'établir dans la vie. Ceci est vrai pour tous les jeunes en situation de précarité, même pour celles et ceux qui s'investissent corps et âme à la poursuite d'une carrière ascendante... En fait, non seulement les salaires ne permettent pas de vivre, mais en plus l'emploi ne remplit pas leur vie. Il est la plupart du temps inintéressant, stressant et dangereux.

Je n'aime pas le travail routine et à l'ouvrage, c'est très routinier. Tous les jours se ressemblent. Je n'ai jamais vu quelque chose d'aussi routinier dans toute ma vie (Charline, 23 ans).

Tu pognais des fatigues dans les épaules et dans les pieds, toujours dans l'humidité, debout sur le ciment. Ils disent que c'est une job, mais ce n'est pas une job pour une personne. C'est une job parce que tu as besoin d'argent (Nathalie, 24 ans).

Avec l'ouvrage que je fais (manutentionnaire), on dirait que tu lèves pesant et dans dix ans de maintenant, tu seras fini (Raoul, 24 ans).

Malgré cela, les jeunes font toutes sortes de travaux, pour la plupart intermittents et mal payés. Ils passent un nombre incalculable d'heures dans des magasins, restaurants, entrepôts et usines, comme journaliers, emballeurs, livreurs, plongeurs, cuisinières, vendeuses, serveuses, etc.

Salariés ou non, ces jeunes réparent des voitures, construisent des maisons, des bijoux, des enseignes; ils coiffent, maquillent, servent à table, travaillent comme auxiliaires de

2. Ils sont $55 \%$ dans notre enquête quantitative à cotiser à l'assurance-chômage. Ce qui signifie, à l'époque, travailler plus de 15 heures/semaine. 
soin, comme aide-enseignants, etc. et, apparemment, le font très bien, et ce, sans avoir appris à l'école les compétences correspondantes de façon formelle ${ }^{3}$. Ce n'est pas par manque de compétence que certains jeunes ne travaillent pas. Comme le confirment ceux qui ont un emploi : «N'importe qui pourrait faire le travail que je fais».

Mais, ces travaux ne les satisfont généralement pas, car ils ne sauvegardent habituellement pas l'essentiel, ils ne sont pas porteurs de sens. Ils symbolisent l'immobilité, tout ce que les jeunes exècrent : la monotonie, la réquisition, la platitude. Tout le contraire du «Go! Go!». Alors comment, à la suite d'un shift épuisant, ne pas avoir l'impression «d'avoir perdu sa journée»?

J'ai des shifts de 11-12 heures par jour. Mon système commence à être habitué. Je n'aime pas d'arriver à l'appartement pour juste me coucher, ce serait perdre ma journée! C'est pour cela que je me couche tard le soir... (Louise, 23 ans).

À quoi bon perdre sa journée, mourir d'ennui ou risquer sa vie au travail... Rien de pire que le sentiment de se donner sans rien acquérir en retour. Certes, ce temps perdu à travailler, ces salaires dérisoires, ces rythmes fatigants, voire dangereux, etc. sont autant de phénomènes qui nourrissent le sentiment d'aliénation, mais il ne faut pas les dissocier de ce qui fait, chez ces jeunes, le mouvement de la vie. Sans quoi, on s'empêcherait de comprendre la portée de ce sentiment d'aliénation qui peut aussi, dans bien des cas et à certains moments, être créateur d'autres équilibres, figures et styles de vie. Ce sentiment d'aliénation se nourrit aussi du rêve hypnotiseur de la consommation.

\section{Des jeunes hypnotisés par la consommation dont ils rêvent d'être délivrés}

Tous ont à se définir par rapport au modèle dominant qui est proposé, comme à nous tous d'ailleurs, à savoir : l'impératif travailler/dépenser qui est devenu la principale maladie des classes moyennes enfermées dans ce que Juliet B. Schor (1991) appelle la «cage d'écureuil» du capitalisme, la débauche des achats faisant fonctionner le système. Les jeunes sont très marqués par ce rêve d'argent et de consommation, notamment les jeunes peu scolarisés qui courent après le travail, par enthousiasme et/ou par nécessité (Grell, 2001). Écoutons l'un d'eux parler de ses 16-17 ans. À présent, il en rit, car il a pris de la distance et a une autre conception de l'existence.

J'aimais l'argent. Les deux dernières années où j'ai été à l'école secondaire, je suivais peutêtre trois cours par année, je travaillais au-dessus de 32 heures par semaine en même temps que mes études. L'argent parle. Demande à n'importe qui, si tu as la chance de faire plus d'argent, que vas-tu faire? Tu vas prendre la job. Même si tu aimes ce que tu fais, tu le lâches... (Jean, 25 ans).

Ces jeunes se mirent de mille façons dans les fantasmes de possession et de calcul. Comme le constate Georg Simmel (1987, p. 411) : «tout ce qui advient aux objets possédés est fonction du sujet qui se déverse en eux». Mais détrompons-nous, cette focalisation

3. Il y a quelques années, se développa un mouvement en faveur de la reconnaissance des acquis... Gageons que si cette politique était prise au sérieux, les employeurs devraient rapidement augmenter les salaires, car ils savent très bien que les jeunes ont toutes les compétences requises pour occuper leurs emplois. 
précoce sur l'argent et les objets de consommation ne dure pas indéfiniment. La plupart du temps, il faut la lire comme une drogue à laquelle le jeune aurait recours pour contrer un état de manque existentiel : une réaction contre le vide (le souci) qui ronge, l'angoissante banalité du monde où les échanges quotidiens sont réduits à des tractations anonymes, dépourvues d'intérêt, utilitaires et commerciales.

Les Compagnies disent qu'elles veulent nous aider... Au fond elle n'en veulent qu'à notre argent (Norbert, 25 ans).

Si les soucis et l'inquiétude, par leur omniprésence, sont des figures de l'existence qui leur sont bien connues, les jeunes, paradoxalement, luttent contre ce sentiment d'aliénation et tentent d'organiser leur vie selon d'autres figures ou modèles (Grell, 2000). Notamment, ces jeunes qui acceptent l'incertitude en accédant à une maîtrise des «soucis», sans toutefois s'en affranchir complètement. Dans d'autres figures, comme celle de la «résistance», on retrouve les jeunes qui, conscients de leur condition, décident de ne pas y céder. Ils se retranchent dans des espaces transitoires qui sont autant de «niches» pour s'abriter, respirer, imaginer des issues et des moyens de traverser (contourner) les épreuves et lieux imposés. Ils sont nombreux à prendre progressivement conscience de leur situation, allant au-devant d'elle, accumulant expérience et savoir-faire tactique (double jeu, rupture, stratégie, multiplication d'espaces, alternance, changement de rythme, etc.). Par exemple, ils ont un «dessein à disposition» (Schutz, 1987, p. 15), des convictions, un idéal moral. Chez eux, la «sédimentation de toutes les expériences humaines antérieures» joue un rôle crucial dans la lutte qu'ils mènent contre le sentiment d'aliénation. Ils rappellent à bon escient que l'existence humaine se fait à plusieurs et que la vie sociale, dont l'interactivité est en quelque sorte la matière première, est d'une «nécessité vitale» pour pouvoir expérimenter les choses par la parole et la discussion avant de s'y engager pour de bon (Zoll, 1992).

J'ai beaucoup changé. Maintenant, je suis vraiment moi-même. Je sépare mon travail de ma vie privée. Ma vie privée, je suis une autre, je suis flyée[...]. Maintenant, je vis. Le temps passe assez vite et je me dis que j'aimerais faire plein de chose! Je suis une personne qui aime rencontrer du monde (Denise, 22 ans).

Maintenant, je vais minimiser mes dépenses. Mon objectif n'est plus de faire de l'argent. Il n'en est plus question. Il est question de ce que j'aime : avoir une vie sociale plutôt que de travailler comme un chien (Jean, 25 ans).

Pour ces jeunes dont la vie est un perpétuel champ de bataille, il faut, à certains moments, sortir de la consommation pour se retrouver, retrouver d'étroites limites sociales et personnelles, inventer d'autres pratiques et significations, pour construire une «petite vie» dont la résistance à l'impératif travailler/dépenser et l'expérimentation d'autres modes de vie sont une raison d'agir.

\section{De nombreux détours : bouger et se repositionner}

Ces jeunes sont très tôt en mouvement. Avec leur zèle et le nombre d'emplois accumulés au cours de leur courte vie, on ne peut qu'être étonné de la ferveur avec laquelle ils se 
lancent dans le travail et la recherche d'emploi. Dès qu'ils en ont la possibilité, ils travaillent et travaillent énormément, ils vont d'un travail à l'autre, de l'école au travail, de Montréal à Vancouver, d'un travail insignifiant aux études, pour pouvoir enfin travailler à ce qu'ils aiment, pour se dépasser, etc. Bien qu'ils traînent avec eux des lacunes scolaires importantes, la grande majorité finissent toujours par améliorer (récupérer) leur scolarité (66\% retournent à l'école). Confrontés à des dangers permanents, ils ont l'attitude du guerrier piégé, de l'innocent injustement emprisonné ou de l'écorché vif, incarnant la lutte du subjectif contre ce qui le corrompt : la vie impossible, la médiocrité étouffante, l'exil de sa propre existence, l'absence de vraie vie. Ils sont vieux avant l'âge et amenés précocement à lutter contre des processus critiques qui semblent, au premier abord, se soustraire totalement à leur contrôle, mais ils sont malgré tout en état, dirait Norbert Elias (1993, p. 77), «d'observer avec une certaine distanciation [...] une solution possible au problème de leur propre vie».

Ils sont généralement très lucides sur la vie qu'ils mènent. Certains se droguent, ils en parlent ouvertement dans cette recherche. D'autres sont dans une impasse : «J'aimerais recommencer ma vie, mais je ne sais pas comment faire». D'autres encore ne planifient rien à l'avance : «Je me lève puis je fais ce qui me tente de faire cette journée-là.» En même temps, on observe que dans leur existence quotidienne, la plupart se montrent capable d'établir des ruptures :

La seule affaire que j'ai faite : je m'ai enjoyé, j'ai bu et pris de la drogue quatre ans de temps. Tout mon argent y est passé... Ça a pris 21 ans avant de commencer à avoir du sens un petit peu [...]. Si tu veux aller prendre une drive en ville, ça prend un joint avant. Si tu veux aller sur le bord de la plage te faire griller, ça prend un joint. Si tu vas rien que faire une tournée de pêche, ça te prend un joint pareille. Si tu veux aller écouter un film, ça te prend un joint. Ben moi, anyway, je suis tanné bien raide. Ça fait déprimer un gars bien tight. Anyway, je trouve ça terrible... Moi, c'est juste cette année que je m’en suis sorti (Luc, 22 ans).

Cette capacité de repositionnement est une grande force. Pour l'amorcer, il faut du temps. Le temps est ici un auxiliaire de l'action. Ces nouvelles orientations, ils ne les prennent pas forcément ni dans le sens ni au moment où, nous, de l'extérieur, nous le voudrions. Pour les comprendre, il faut accepter d'y regarder de près, loin des poncifs qu'égrène l'idéologie dominante. Même dans l'univers que le discours officiel caractérise de «culture de la dépendance», on constate que les jeunes bougent, construisent leur vie, font preuve d'imagination. Prenons, par exemple, une des cibles privilégiées des apôtres de cette culture, à savoir : les jeunes femmes issues de milieux défavorisés, sortant de l'école sans formation (décrochage, cours pratiques, etc.), qui ont vécu une grossesse précoce, et finissent inévitablement sur l'aide sociale. Elles sont le portrait type de tous les comportements que stigmatise cette fameuse culture de la dépendance, déterminé à n'y voir qu'une population à risque. Or, à travers les récits de l'enquête, quand on considère l'intelligence que ces jeunes femmes mettent à faire leur vie, à la construire, on ne peut qu'être émerveillé de leur capacité à correspondre à «l'écoute de l'offre du moment»(Grell, 1999, p. 220), c'est-à-dire : leur habileté à saisir les occasions, à cueillir les opportunités de formation, à mêler l'utile et l'imaginatif, les joies de 
la vie, etc... pour dépasser (transcender) les situations auxquelles elles sont confrontées. Ces compétences tactiques ont été exposées avec la plus grande vigueur dans Cosmos de Witold Gombrowicz, quand il fait parler, son héros qui répète à qui veut l'entendre :

J'ai dû, vous le comprenez, recourir toujours davantage à de tout petits plaisirs, presque invisibles, des à-côtés... Vous n'avez pas idée combien, avec ces petits détails, on devient immense, c'est incroyable comme on grandit (1965, p. 139-140).

Dans ce cosmos, ce «monde plénier» qu'est le monde humain créé par l'homme, l'imagination créatrice (le sens figuré) ne vient nullement se substituer au manque, à l'aliénation d'une vie qui ne serait que survivance. Elle vient agir, faire "grandir», constituer un équilibre entre le désir d'une «vraie vie» et les assignations mortifères du climat ambiant. Chez ces jeunes, le mouvement — bouger, alterner, se repositionner est vital. Une façon, bien à eux, de lutter contre le sentiment de perdre son temps et sa jeunesse à l'école, au travail et dans d'autres lieux formalisés. Le mouvement est aussi largement initiatique comme moyen d'apprendre à faire sa vie malgré l'école qui est «plate», à gagner son pain sans être rongé par la fatigue du travail, à fumer sans «tomber dans la drogue», etc. Dans ce va-et-vient continuel (rituel), ils accumulent des résidus dissonants qui les situent dans d'autres lieux et permettent de contourner plus facilement les déterminations qui pèsent sur leur vie.

\section{D'autres formes de résistance à l'œuvre pour faire leur vie}

Leurs résistances s'organisent en trois grandes sphères. Dans la première, se regroupent celles qui, face à des difficultés incontrôlables, s'accompagnent d'une forte imprégnation affective. Lorsque le monde qui vous entoure apparaît comme insaisissable, l'audace de l'amour, la passion et l'excès, sont plus réalistes qu'un niveau élevé de prudence ou de programmations anticipatrices.

Avant on se disait : «plus tard, on va faire ceci, on va faire ça». Je me dis maintenant qu'il ne faut jamais dire à plus loin. Maintenant, $j$ 'ai à dire que ça va aller comme ça va aller. Je ne fais plus de plan. L'essentiel, pour moi, c'est que sans amour je me chavirerais. Il faut qu'une personne montre à l'autre qu'elle l'aime. C'est pas une vie d'être là, sans petits mots doux (Colette, 22 ans).

Ici, domine la capacité à vivre le temps présent et l'excès : s'éclater, se passionner, se défoncer, jouer un autre personnage, vivre intensément, etc. avec un souci évident d'idéalité et de perfection qui dénote un niveau élevé d'attentes et d'exigences. Flaubert ne disait-il pas : «L'excès est une preuve d'idéalité» qui pousse à «aller au-delà du besoin »? C’est ce niveau élevé d'idéalité qui explique chez les jeunes, le côté démesuré et passionné de leurs implications de tous ordres et de toutes espèces (amour, amitié, travail, moto, boisson, drogue, etc.). Lorsque aimer atteint les apparences de l'idiotie et danser celles de la transe, lorsque fumer de la drogue se transforme en rituel quotidien, que la moto se métamorphose en drogue et la chasse en une ode à la nature, etc., nous sommes au-delà de la nécessité et de plain-pied dans le sentiment de lutte contre l'aliénation. 
La deuxième sphère regroupe les résistances qui se fondent plus sur la conjoncture et l'occasion que sur l'affectivité et l'amour. Ce qui demande deux conditions de réalisation : d'une part, un art subtil de la tactique; d'autre part, un monde laissant des ouvertures suffisantes aux jeunes, car le hasard n'a rien à offrir dans un monde aux issues bouchées. Dans cette sphère, nous avons la capacité de contrôler les fantasmes de miracle, celle de saisir l'offre du moment et les opportunités qui se présentent.

La troisième sphère s'adresse aux jeunes qui ont la chance de maîtriser, du moins en partie, certains aspects du processus critique dans lequel se trouve leur existence. Ils sont en position de développer des stratégies et de concrétiser des projets. Ils ont, par exemple, la capacité de domestiquer l'incertitude permanente et de développer de multiples activités.

Trop avoir, ce n'est pas bon. Moi, ce que je veux c'est une petite vie. Que je puisse vivre un petit peu avec ma femme, les gens que j'aime, m’occuper de mes légumes, couper du bois... (Roger, 24 ans).

\section{LUTTER CONTRE LE «SENTIMENT D'ALIÉNATION 》}

Pour des jeunes, avec une scolarité faible en périphérie du travail salarié et des grands centres socioéconomiques, se pose la question de l'insignifiance d'un monde qui ne constitue plus une armature existentielle suffisante. Immergés dans un espace-temps donné, ces jeunes font très tôt l'expérience de processus contre lesquels se frappent nombre de leurs actions, discours et sentiments. Ils sont très tôt plongés dans des situations, institutions et représentations qui altèrent et entravent le déploiement de leur vie et celle des groupes auxquels ils appartiennent. Tant dans leur vie diurne que dans leur vie nocturne, en famille, au travail, à l'école, dans la rue, ils sont confrontés aux contraintes émotionnelles et morales, aux assignations hiérarchiques et pratiques, aux injonctions contradictoires d'une société pseudo-démocratique asservie à la loi du marché et à la concurrence sans pitié entre individus : il leur faut aimer la servitude, se fondre avec passion dans des objets de consommation, adopter «librement» des attitudes de soumission, faire semblant d'être bien dans leur peau, satisfaire les "petits chefs» de tous poils et de toute espèce, se débrouiller pour répondre aux attentes, se couler dans les différents moules (école, travail, consommation, etc.) d'une société dont le pouvoir s'appuie de plus en plus sur l'offense, l'allégeance et la richesse. Malmenés, manipulés, illusionnés, la plupart de ces jeunes sont projetés à toute vitesse. «La machine a éjecté son conducteur et elle file à l'aveuglette à travers les espaces » (Horkheimer, 1974, p. 137). Ces jeunes sont confrontés en permanence à des processus critiques : des passages à vide et des épreuves. Et cela, le plus naturellement du monde, comme si ces processus étaient inévitablement extérieurs, étrangers, le résultat de «lois extrahumaines», dirait Polanyi.

Ce climat général d'incertitude pour le moins négatif (angoissant) crée tôt chez eux le sentiment d'être nés «du mauvais côté», «dans le mauvais monde» ou «au bas de l'échelle»; là où la vie est d'office mortelle, espace emmuré, temporalité terne et monotone. Dès l'âge de 15-16 ans, ces jeunes font l'expérience, par exemple, que le travail ne 
possède aucune valeur expressive, qu’il faut des «connexions» pour avoir un emploi, que les «modifiés sont des losers», que "ceux des cours pratiques sont comme mis à part», etc. Ce sont généralement les lieux plus formalisés comme l'école et le travail qui se chargent de le leur signifier, à travers toute une série d'épreuves perçues par eux comme des non-lieux — «l'école, ce n'est pas pour moi», «travailler comme un chien», «ce n'est pas un travail pour une personne» —, mais qui, en fait, métamorphosent petit à petit les intimations sociales en responsabilités individuelles (Grell, 1999).

À l'intérieur de ce climat général, les jeunes tentent de sauver l'essentiel : une espérance vivante envers et contre ce monde mortifère. Tout au long de ce trajet, ils font la distinction entre une vie "dévalorisée», sans qualité ni sens, au milieu d'un monde inconsistant (déboussolé), et une existence "digne d'être vécue», "valorisée», dans une société qui accueillerait ses membres en les aidant à s'orienter. Mais, pour eux, cette société se définit d'abord et avant tout par l'absence, le manque, le mépris de toutes ces choses (choses qui, dans certains cas, sont immatérielles, par exemple la dignité, l'amour, la fidélité, etc.) dont la «vraie vie» est directement tributaire et dont l'accessibilité, en quantité suffisante et selon des modalités adéquates, constitue une condition nécessaire. Il s'ensuit pour nombre d'entre eux des formes variées de résistance et tout un travail de refondation des pratiques tournant autour du comment habiter ce monde, voire comment le transformer, pour y faire sa vie.

Cette question en appelle immédiatement une autre, à savoir : comment lutter contre le sentiment d'aliénation? Ce sentiment global d'ordre idéologique qui, nous l'avons vu, s'enracine dans de nombreuses expériences de rupture - concrètes et sensibles : en famille, à l'école, au travail, dans la vie en général - et dont les effets de conscience créent un procès continuel par lequel ces jeunes attestent une certaine reprise en main de leur propre vie et les guident dans des actions, situations, modes de vie qui, loin de leur aliéner les images et conditionnements déjà acquis, les intègrent dans une réalité nouvelle. Pour combattre ce sentiment d'aliénation aux bases empiriques certaines, ces jeunes vont utiliser différents stratagèmes, parmi lesquels on trouve des mouvements de va-et-vient et de repositionnement, la création d'espaces intermédiaires, des glissements de significations et substitutions de sens, des transferts de compétence, etc. Ce sont là autant de tentatives de dépassement des rapports rompus (ou inexistants) entre eux et le monde. Ce sont des essais de réconciliation avec les choses, les activités et les institutions, car ils ne savent pas vivre seulement en fonction d'elles, mais pour des opinions, des convictions, des sentiments, «pour ce lien imaginaire et secret qui lie et relie le monde et les choses au cour de la conscience» (Durand, 1984, p. 501).

Ce travail de réconciliation, sur un territoire où ils cherchent à vivre à peu près, est un travail ardu dont les œuvres puisent abondamment dans les représentations, les images, les jeux d'esquives, les scènes de la vie quotidienne, les formes et figures de l'imaginaire social. Il s'agit, pour ces jeunes, de trouver un moyen de construire et de sauvegarder leur jardin secret, des zones d'autonomie dont ils sont maîtres. Tel est l'enjeu et l'explication de la duplicité sociale, voire de la malice, avec laquelle plusieurs 
abordent en toute conscience les conventions du monde par l'expression d'une normalité d'apparence. Ils travailleront, par exemple, pendant quelques années dans un grand magasin en donnant l'impression d'être contents de leur emploi, alors qu'ils le trouvent vraiment «plate» :

Je voulais seulement rester là tant que je pouvais pour montrer que j'étais capable de rester à une place vraiment longtemps [...]. Je suis aussi bien de dire que j'aime ça parce que je ne peux pas dire que je ne l'aime pas. Je suis obligé de prendre ça comme ça vient (Rick, 23 ans).

Cette apparente soumission au travail masque le plus souvent un écart véritable (un art de l'arrangement) traduisant une multiplicité d'îlots refuges qui sont autant d'espaces leur permettant d'échapper et de garder une certaine intégrité. En fait, dans la distance qu'ils établissent par rapport à leur travail officiel se logent de multiples espaces de libre activité, dont le travail qu'ils effectuent pour eux-mêmes, par exemple, en réparant des moteurs ou en restaurant leur maison ou celle de quelqu'un d'autre.

Ces jeunes se comportent en acteurs, souvent géniaux, d'une pièce de théâtre qui n'est pas la leur. Ils construisent un «ailleurs» où ils réfugient. En rusant, ils se donnent les moyens de ressentir profondément qu'ils ne sont pas inexorablement prisonniers des lieux et espaces imposés, ils se donnent des arguments pour réfuter ce «dualisme pessimiste» dont parle si bien Raymond Ledrut : «ce dualisme qui nous engage à supporter la réalité puisque l'idéal n'arrive jamais à entrer durablement dans le réel» (Ledrut, 1979, p. 99).

Telle une exigence sans illusions, ils sont nombreux à lutter contre le sentiment d'aliénation et à se débattre pour vivre à peu près correctement dans un monde précaire et sans compassion. Vivre à peu près n'est pas vivre à moitié, au quart ou au trois quarts, mais renvoie à un vécu d'ordre global. C'est être au monde en tentant d'incarner les promesses de liberté, d'autonomie, de développement qui caractérisent, dans les sociétés occidentales, une vie pleinement humaine, alors même que la vie qui les travaille entrave leur capacité d'autoproduction et d'auto-organisation. Loin des sentiers de l'idéologie néolibérale qui affirme que tout est possible pour tous, mais néanmoins extrêmement au fait des mécanismes économiques et sociaux à l'œuvre dans leur région, ces jeunes contribuent à construire nos façons d'être ensemble. Car, à travers des itinéraires singuliers — leur «passage au monde précaire» — se construisent aussi des processus sociaux de réappropriation de l'existence. 


\section{AN NEXE \\ Informations sur l'enquête au départ de cette réflexion}

Cette enquête s'effectua entre 1993 et 1995, et porte sur les jeunes francophones de l'est du Nouveau-Brunswick qui ont eu une scolarité faible au cours de leurs études secondaires. La population étudiée $(\mathrm{n}=2,780)$ comprend tous les jeunes qui ont fréquenté, au cours des années 1988-1991, une des 12 écoles polyvalentes de l'est du NouveauBrunswick, et qui ont pris au moins deux cours de niveau modifié en français et/ou mathématique. L'échantillon de départ est statistiquement représentatif $(n=415)$. L'enquête s'effectua en deux étapes, avec un intervalle d'un an entre les deux.

La première étape comportait un questionnaire et des entrevues semi-dirigées ayant pour fonction d'établir un portrait factuel des jeunes peu scolarisés : différentes configurations de parcours scolaire et de travail, mais aussi l'évolution de leurs situations affectives (amours, amitiés), leur consommations de drogues et leur expérience de certaines formes de violence, pour enfin établir leur portrait familial. Le taux de participation fut de $66 \%$.

La deuxième étape comportait des récits de vie $(\mathrm{n}=37)$ qui avaient pour fonction d'approfondir, à partir des informations recueillies dans la première étape, la mosaïque sociale du passage au monde précaire. À cette étape, on a procédé à un entretien biographique court et ciblé (de type récit de pratique) d'une durée de deux heures et demi environ, dont la consigne portait sur trois aspects de la biographie : les repères marquants de la trajectoire, le passage au monde adulte et le rapport au travail.

\section{RÉSUMÉ}

L'auteur propose une lecture du sentiment d'aliénation en tant qu'opérateur de mouvance. II appuie sa réflexion sur les récits d'expérience de jeunes en situation précaire dont il présente à grands traits le portrait vivant, filmé, de leurs confrontations avec le monde précaire qui les entoure. Dans la perspective adoptée, il s'agit d'atteindre la base symbolique des pratiques, avec, notamment, les détours que ces jeunes prennent pour vivre et agir. Parler de sentiment d'aliénation à leur propos participe d'une attitude notionnelle qui procède par approches concentriques et prend acte des conditions et expériences auxquelles nombre de leurs actions, discours et sentiments sont confrontés. Ils font face en permanence à des processus critiques : des passages à vide et des épreuves. Et cela, le plus naturellement du monde, comme si ces processus étaient inévitablement extérieurs, étrangers, le résultat de lois extrahumaines. Pourtant, ces jeunes résistent et tentent de sauver l'essentiel.

\section{SUM MARY}

The author proposes a reading of the sense of alienation as a form of mouvance. His reflection is based on the experiences of youth in a situation of precariousness which he presents openly as a living portrait, taken from their confrontations with the precarious world in which they live. In this perspective, one must attain the practical symbolic foundations, namely the detours that these youth must take in order to live and react. To speak about their sense of alienation, one must 
evoke a notional attitude that proceeds by way of concentric approaches and takes note of the conditions and experiences in which many of their actions, discourse and feelings are torn apart. They are permanently facing critical processes : meaningless actions and ordeals. And that, as the most natural thing in the world, as if these processes were alien, foreign to them, the result of super-human laws. However, these youth resist and try to save the essential.

\section{RESUMEN}

El autor propone una lectura del sentimiento de alienación como que operador de influencia. Él funda su reflexión sobre los relatos de vivencias de jóvenes en situación precaria del que presenta a grandes rasgos el retrato vivo, filmado, de sus confrontaciones con el mundo precario que los rodea. En la perspectiva que él adopta, se trata de alcanzar la base simbólica de las prácticas, principalmente con los rodeos que estos jóvenes hacen para vivir y actuar. Hablar del sentimiento de alienación, a este propósito, parte de una actitud nocional que procede por aproximaciones concéntricas y da cuenta de las condiciones y experiencias de las cuales se desprenden numerosas de sus acciones, discursos y sentimientos. Ellos se enfrentan permanentemente con procesos críticos : malos momentos y pruebas. Y ésto como lo más natural del mundo, como si estos procesos fueran inevitablemente exteriores, ajenos, el resultado de leyes extra-humanas. Sin embargo, estos jóvenes resisten e intentan salvar lo esencial.

\section{BIBLIOGRAPHIE}

Arendt, H. (1983), Condition de l'homme moderne, Paris, Calmann-Lévy.

Berthelot, J.-M. (1990), L'intelligence du social, Paris, Presses Universitaires de France.

Blin, T. (1999), Phénoménologie de l'action sociale, Paris, L'Harmattan.

Castoriadis, C. (1975), L'institution imaginaire de la société, Paris, Seuil.

Chaumont, J.-M. (1991), Autour d'Auschwitz. De la critique de la modernité à l'assomption de la responsabilité historique : une lecture de Hannah Arendt, Bruxelles, Académie royale de Belgique.

Cingolani, P. (1994), «L'interminable pérégrination... », Tumultes, nº 5, p. 95-122.

Cingolani, P. (1986), L'exil du précaire. Récits de vie en marge du travail, Paris, Méridiens Klincksieck.

Dumont, F. (dir.) (1986), Une société des jeunes?, Québec, Institut québécois de recherche sur la culture.

Durand, G. (1984), Les structures anthropologiques de l'imaginaire, Paris, Dunod.

Elias, N. (1993), Engagement et distanciation, Paris, Fayard.

Elias, N. (1991), La société des individus, Paris, Fayard.

Galland, O. (1990), "Un nouvel âge de la vie», Revue française de sociologie, vol. 31, p. 529-551.

Galland, O. (1984), «Précarité et entrées dans la vie», Revue française de sociologie, vol. 25, p. 49-66.

Gauthier, M. (1996), «Le marché du travail comme lieu de construction des marges chez les jeunes», Cahiers de recherche sociologique, no 27, p.17-29.

Gauthier, M. (1994), Une société sans les jeunes?, Québec, Institut québécois de recherche sur la culture. Gauthier, M. et L. Mercier (1994), La pauvreté chez les jeunes. Précarité économique et fragilité sociale, Québec, Institut québécois de recherche sur la culture.

Geertz, C. (1986), Savoir local, savoir global : les lieux du savoir, Paris, Presses Universitaires de France.

Gombrowicz, W. (1965), Cosmos, Paris, Denoël.

Gorz, A. (1997), Misères du présent. Richesse du possible, Paris, Éditions Galilée.

Grell, P. (2001), «Sur les conditions d'existence des jeunes dans un monde précaire», Sociétés.

Grell, P. (2000), «Le sentiment de l'existence : recherche auprès de jeunes précaires», Possibles, vol. 24, $\mathrm{n}^{\text {os } 2-3, ~ p . ~ 209-228 . ~}$

Grell, P. (1999), Les jeunes face à un monde précaire. Récits de vie en périphérie des grands centres, Paris, L'Harmattan. 
GRELL, P. (1985), Études du chômage et de ses conséquences : les catégories sociales touchées par le non-travail, Groupe d'analyse des politiques sociales, Université de Montréal.

Grell, P. et A. Wery (1993), Héros obscurs de la précarité. Récits de pratiques et stratégies de connaissance, Paris, L'Harmattan.

Horkheimer, M. (1974), Éclipse de la raison, Paris, Payot.

LEDRut, R. (1979), La révolution cachée, Bruxelles, Casterman.

LEFort, C. (1955), «L'aliénation comme concept sociologique», Cahiers internationaux de sociologie, janv.juin 1955 , p. 35-54.

Maffesoli, M. (1985), La connaissance ordinaire. Précis de sociologie compréhensive, Paris, Librairie des Méridiens.

Marcuse, H. (1968), L’homme unidimensionnel, Paris, Les Éditions de Minuit.

PACOM, D. (1999), «Hommage à Cornelius Castoriadis», Possibles, vol. 23, nº 2, p. 175-205.

Polanyi, K. (1983), La grande transformation, Paris, Éditions Gallimard.

RenÉ, J.-F. (1993), «La jeunesse en mutation : d'un temps social à un espace social précaire», Sociologie et société, vol. $25, \mathrm{n}^{\mathrm{o}} 1, \mathrm{p} .153-171$.

Riceur, P. (1974), «Aliénation», Encyclopaedia Universalis, vol 1, p. 660-664.

SChnApper, D. (1989), «Rapport à l'emploi, protection sociale et statuts sociaux ", Revue française de sociologie, vol. 30, p. 3-29.

Schenr, S. (2000), «Processus de singularisation et formes de socialisation de la jeunesse», Lien social et politiques - RIAC, $\mathrm{n}^{\circ} 43$, p. 49-58.

Schenr, S. (1999), La vie quotidienne des jeunes chômeurs, Paris, Presses Universitaires de France.

ScHor, J. (1991), The overworked american. The unexpected decline of leisure, New York, Basic Books.

Schutz, A. (1998), Éléments de sociologie phénoménologique, Paris, L'Harmattan.

Schutz, A. (1987), Le chercheur et le quotidien. Phénoménologie des sciences sociales, Paris, Librairie des Méridiens.

Seeman, M., D. Vidal, M. Amiot et A. Touraine (1967), «Débat : de l'utilité sociologique de la notion d'aliénation", Sociologie du travail, $\mathrm{n}^{\circ}$ 2, p. 180-209.

Simmel, G. (1987), Philosophie de l'argent, Paris, Presses Universitaires de France.

Singly, F. de (2000), «Penser autrement la jeunesse», Lien social et politiques - RIAC, $\mathrm{n}^{\circ}$ 43, p. 9-21.

VIDAL, D. (1969), «Un cas de faux concept : la notion d'aliénation», Sociologie du travail, nº 1, p. 61-82.

Weber, M. (1967), L'éthique protestante et l'esprit du capitalisme, Paris, Plon.

Zoll, R. (1992), Nouvel individualisme et solidarité quotidienne, Paris, Éditions Kimé. 